\title{
Adalimumab in active ulcerative colitis: A "real-life" observational study
}

\author{
Italian Group for the Study of Inflammatory Bowel Disease, Alessandro Armuzzi ${ }^{\mathrm{a}, *}$, Livia Biancone ${ }^{\mathrm{b}}$, \\ Marco Daperno $^{b}$, Alessandra Coli ${ }^{b}$, Daniela Pugliese ${ }^{a}$, Vito Annese ${ }^{b}$, Annalisa Aratari $^{b}$, \\ Sandro Ardizzone ${ }^{\mathrm{b}}$, Paola Balestrieri ${ }^{\mathrm{b}}$, Fabrizio Bossa ${ }^{\mathrm{b}}$, Maria Cappello ${ }^{\mathrm{b}}$, Fabiana Castiglione ${ }^{\mathrm{b}}$, \\ Michele Cicala $^{b}$, Silvio Danese ${ }^{\mathrm{b}}$, Renata D'Incà ${ }^{\mathrm{b}}$, Pietro Dulbecco ${ }^{\mathrm{b}}$, Giuseppe Feliciangeli ${ }^{\mathrm{b}}$, Walter Fries ${ }^{\mathrm{b}}$ \\ , Stefania Genise ${ }^{\mathrm{b}}$, Paolo Gionchetti ${ }^{\mathrm{b}}$, Stefano Gozzi ${ }^{\mathrm{b}}$, Anna Kohn ${ }^{\mathrm{b}}$, Roberto Lorenzetti ${ }^{\mathrm{b}}$, Monica Milla ${ }^{\mathrm{b}}$, \\ Sara Onali ${ }^{\mathrm{b}}$, Ambrogio Orlando $^{\mathrm{b}}$, Luigi Giovanni Papparella $^{\mathrm{b}}$, Sara Renna ${ }^{\mathrm{b}}$, Chiara Ricci $^{\mathrm{b}}$, \\ Fernando Rizzello $^{\text {b }}$, Raffaello Sostegni ${ }^{\text {b }}$, Luisa Guidi ${ }^{\text {a }}$, Claudio Papi ${ }^{\text {b }}$
}

a Internal Medicine and Gastroenterology Unit, Complesso Integrato Columbus, Catholic University, Rome, Italy

${ }^{\mathrm{b}}$ See Appendix A

\section{A R T I C L E I N F O}

\section{Article history:}

Received 30 November 2012

Accepted 24 March 2013

Available online 15 May 2013

\section{Keywords:}

Adalimumab

"Real-life" study

Ulcerative colitis

\begin{abstract}
A B S T R A C T
Background and aims: The effectiveness of adalimumab in the treatment of ulcerative colitis is under debate. Although controlled trials have shown that adalimumab is significantly better than placebo, the absolute clinical benefit is modest. We report data on the effectiveness of adalimumab in a cohort of ulcerative colitis patients treated in 22 Italian centres.

Methods: All patients with active disease treated with adalimumab were retrospectively reviewed. Coprimary endpoints were clinical remission at weeks 4, 12, 24 and 54. Secondary endpoints were sustained clinical remission, steroid discontinuation, endoscopic remission and need for colectomy.

Results: Eighty-eight patients were included. Most patients had received previous infliximab treatment. Clinical remission rates were $17 \%, 28.4 \%, 36.4 \%$ and $43.2 \%$ at $4,12,24$ and 54 weeks respectively. Twentytwo patients required colectomy. Clinical remission and low C-reactive protein at week 12 predicted clinical remission at week 54 (OR 4.17, 95\% CI 2.36-19.44; OR 2.63, 95\% CI 2.32-14.94, respectively). Previous immunosuppressant use was associated with a lower probability of clinical remission at week 54 (OR 0.67, 95\% CI 0.08-0.66) and with a higher rate of colectomy (HR 9.7, 95\% CI 1.46-9.07).

Conclusion: In this large "real-life" experience adalimumab appears effective in patients with otherwise medically refractory ulcerative colitis. Patients achieving early remission can expect a better long-term outcome.
\end{abstract}

(c) 2013 Editrice Gastroenterologica Italiana S.r.l. Published by Elsevier Ltd. All rights reserved.

\section{Introduction}

Ulcerative colitis (UC) is an idiopathic, chronic inflammatory condition of the large intestine, characterised by a relapsing and remitting course. The disease has a lifelong clinical course and can have a significant negative impact on the patient's quality of life [1]. The main goal of therapy in UC is to induce and maintain clinical remission: although conventional medical therapies, including 5-aminosalicylic acid, corticosteroids and immunosuppressants (azathioprine, 6-mercaptopurine and cyclosporine) are effective in the majority of patients, in a substantial proportion these agents inadequately control the disease or can lead to adverse

\footnotetext{
* Corresponding author at: Internal Medicine and Gastroenterology Unit, Complesso Integrato Columbus, Catholic University, Via G. Moscati 31, 00168 Rome, Italy. Tel.: +3906 3503310; fax: +39063054641.
}

events. Therefore, new therapies, beyond conventional treatment, are needed for many patients with UC [2].

Over the last few years, major advances in the understanding of the pathogenesis and natural course of disease have substantially increased the therapeutic options in UC. Tumour necrosis factor alpha (TNF $\alpha$ ) is an important cytokine involved in the pathogenesis of inflammatory bowel disease and targeting this cytokine may be an effective therapy in patients with UC [3]. Infliximab, a chimeric monoclonal antibody to TNF $\alpha$ has been shown to be effective for inducing and maintaining response and remission, inducing mucosal healing and reducing the need for colectomy in patients with moderate-to-severe UC that is refractory to conventional treatment [4-9]. However, infliximab is immunogenic and infusion reactions and loss of response related to antibodies to infliximab may be a relevant problem [10].

Adalimumab (ADA) is a fully human anti-TNF $\alpha$ monoclonal antibody that does not share immunogenicity with infliximab. ADA consists of human-derived heavy and light chain variable regions 
and a human IgG1 constant region: it binds specifically to TNF $\alpha$ and blocks its interaction with the $\mathrm{p} 55$ and $\mathrm{p} 75$ cell surface TNF receptors [11]. ADA has been shown to be effective and safe for inducing and maintaining remission in patients with moderate to severe Crohn's disease (CD), either naïve to anti-TNF $\alpha$ or with previous loss of response or intolerance to infliximab [9,12-15]. Open-label and retrospective studies have shown that ADA can be an effective therapeutic option for inducing and maintaining remission in patients with active UC refractory or who are intolerant to standard therapy [16-21].

Recently, two randomised controlled trials (RCTs) have shown that ADA is more effective than placebo for inducing and maintaining remission in patients with moderate-to-severe UC who did not have an adequate response to conventional therapy, including steroids and immunosuppressants [22,23]. However, the absolute benefit is not impressive and this has been a matter of some debate. The present study reports data on the short- and long-term effectiveness and safety of ADA in a large, multicentre cohort of UC patients treated in 22 Italian IBD referral centres.

\section{Materials and methods}

This study consisted of an uncontrolled, open-label retrospective case series of UC patients treated with ADA in different Italian IBD referral centres. Eligible patients included men and women at least 18 years of age with an established diagnosis of UC according to standard criteria [24]. All patients were classified according to the "Montreal Classification" [25] and had to have active disease, defined as a partial Mayo score $\geq 2$ points [4,26], despite concomitant treatment. ADA induction and maintenance regimen, the need for dose escalation and timing of treatment discontinuation were left to the investigators' judgement, as well as concomitant medications including oral and topical aminosalicylates, steroids and immunosuppressants. A shared common database was used to collect demographic and clinical data. Data collected at baseline were: gender, age at diagnosis, disease extension, disease duration, smoking habits, previous immunosuppressive and infliximab therapies, concomitant medications at baseline, CRP levels, partial Mayo score and Mayo endoscopic subscore. Patients were clinically assessed at weeks 4 and 12 and thereafter at weeks 24 and 54. Partial Mayo score, C-reactive protein (CRP) levels, ADA maintenance scheme, prednisone equivalents, and adverse events were recorded at each time point.

The co-primary endpoints were clinical remission at different time points: 4, 12, 24 and 54 weeks. The secondary endpoints included: (1) sustained clinical remission; (2) steroid-sparing effect; (3) endoscopic remission; (4) need for colectomy. Clinical remission was defined as a partial Mayo score $\leq 1$ : sustained clinical remission was arbitrarily defined as clinical remission at week 12 maintained through weeks 24 and 54. Endoscopic remission was defined as a Mayo endoscopic subscore $\leq 1$. A corticosteroid-sparing effect was defined as corticosteroid discontinuation without recurrence of symptoms, in patients receiving corticosteroids at baseline.

The intention-to-treat (ITT) population included all patients who had received at least one course of ADA. The percentage of patients in clinical remission at different time points was calculated according to ITT analysis. Statistical analysis of remission rates at different time points was limited to descriptive statistics. Continuous variables were described using medians with interquartile range (IQR). Kaplan-Meier survival curves were used to assess the cumulative probability of continuing ADA during follow-up and to assess the cumulative probability of colectomy for treatment failure or intolerance. Univariate analysis was performed to assess the possible influence of clinical variables on clinical remission at 54 weeks. Categorical variables were analysed using the Chi-squared test. Odds ratio (OR) was given with $95 \%$ confidence intervals (95\%
$\mathrm{CI})$ and two-sided $p$ values. A $p$ value $<0.05$ was considered statistically significant. The following covariates were considered: sex, age, disease duration, main indications for ADA (corticosteroid resistance or dependence), previous anti-TNF $\alpha$ exposure, previous immunosuppressant use, corticosteroid therapy at baseline, clinical remission and CRP level at 12 weeks. Parameters with a $p$ value less than 0.05 using univariate analysis were entered into a multivariate logistic regression model to identify independent predictors for clinical remission at 54 weeks. To look for predictive factors of colectomy, univariate analysis with log-rank test was used considering the following covariates: sex, age, disease duration and extension, previous anti-TNF $\alpha$ exposure, main indications for ADA (corticosteroid resistance or dependence), previous immunosuppressant use, corticosteroid therapy at baseline and baseline CRP levels. Hazard ratio (HR) was given with 95\% confidence intervals $(95 \% \mathrm{CI})$. All variables with a $p$ value less than 0.05 at log-rank test were entered into a Cox proportional hazards survival regression.

One way analysis of variance (ANOVA) was used to assess differences among CRP levels, daily prednisone equivalents and partial Mayo score values at different time points. When significant differences ( $p$ value $<0.05$ ) emerged from ANOVA, post hoc pairwise comparison of subgroups, using Student-Newman-Keuls test, was made.

Analyses were performed using MedCalc ${ }^{\circledR}$ (Version 9.3.7.0) and Epistat (copyright @ Epistat Services, 1991).

\section{Results}

\subsection{Baseline patient characteristics}

From April 2006 to July 2011, 88 active UC patients (35 male and 53 female), with a median age at diagnosis of 27.7 years (IQR 19.8-35.9) were enrolled. Patients were treated in 22 Italian IBD referral centres. Fifty-seven patients (64.8\%) had extensive colitis, 27 (30.7\%) had left-sided colitis and 4 (4.5\%) had proctitis. The median duration of disease was 8.9 years (IQR 4.5-15.3). The vast majority of patients were non-smokers or ex-smokers and only 7 patients $(7.9 \%)$ were active smokers. The indications for ADA treatment were corticosteroid dependence in 41 patients (46.6\%), corticosteroid resistance in 23 patients (26.1\%), extra-intestinal manifestations in 14 patients (15.9\%) and a combination of corticosteroid dependence and extra-intestinal manifestations in 10 patients (11.4\%).

Sixty-nine patients (78.4\%) were previously treated with infliximab and only 19 patients (21.6\%) were naïve to anti-TNF $\alpha$. Among the former group, the median duration of infliximab therapy was 11.0 months (IQR 4.7-18.0), with a median number of infusions per patient of 8.0 (IQR 4.7-12.2). The main reasons for infliximab discontinuation were primary non-response in 5 patients $(7.2 \%)$, loss of response in 27 patients (39.1\%), intolerance in 23 patients (33.3\%), loss of response and intolerance in 3 patients (4.3\%), infections in 3 patients $(4.3 \%)$ and other causes in 8 patients $(11.6 \%)$. The median time from the end of infliximab to the start of ADA therapy was 2.07 months (IQR 1.0-13.2).

Sixty-five patients (73.9\%) were previously treated with immunosuppressants: 49 with thiopurines, 7 with methotrexate, 1 with cyclosporine, 3 with thiopurines and methotrexate, 4 with thiopurines and cyclosporine, 1 with cyclosporine, thiopurines and methotrexate.

At baseline, the median partial Mayo score was 6 (IQR 4-8), with no significant differences between infliximab-naïve and infliximab-exposed patients. Eighty-four patients (95.4\%) underwent endoscopy at enrolment and the median Mayo endoscopic subscore was 2.0 (IQR 2-3). The median CRP serum level was $10.95 \mathrm{mg} / \mathrm{L}$ (IQR 4.85-19.6) and raised CRP serum levels were 
Table 1

Patients' characteristics at baseline.

\begin{tabular}{|c|c|}
\hline Patients & Total number 88 \\
\hline Male, $n(\%)$ & $35(39.8)$ \\
\hline Age at diagnosis, years, median (IQR) & $27.7(19.8-35.9)$ \\
\hline $\begin{array}{l}\text { Duration of disease, years, median } \\
\text { (IQR) }\end{array}$ & $8.97(4.5-15.3)$ \\
\hline Extension of disease - E1, E2, E3, $n(\%)$ & 4 (4.5), 27 (30.7), 57 (64.8) \\
\hline Previous infliximab, $n(\%)$ & $69(78.4)$ \\
\hline $\begin{array}{l}\text { Previous immunosuppressants } \\
\text { (AZA/6MP, MTX, Cya) }{ }^{\mathrm{a}}, n(\%)\end{array}$ & $65(73.9)$ \\
\hline Concomitant corticosteroids, $n(\%)$ & $60(68.1)$ \\
\hline \multicolumn{2}{|l|}{ Main indication to adalimumab, $n(\%)$} \\
\hline Corticosteroid resistance & $23(26.1)$ \\
\hline Corticosteroid dependence & $41(46.6)$ \\
\hline EIMs $^{\mathrm{b}}$ & $14(15.9)$ \\
\hline Corticosteroid dependence + EIMs & $10(11.4)$ \\
\hline Partial Mayo score, median (IQR) & $6.0(4-8)$ \\
\hline $\mathrm{CRP}^{\mathrm{C}}(\mathrm{mg} / \mathrm{L})$, median $(\mathrm{IQR})$ & $10.9(4.9-19.6)$ \\
\hline \multicolumn{2}{|l|}{ Adalimumab induction regimen, $n(\%)$} \\
\hline $160 / 80 \mathrm{mg}$ & $77(87.5)$ \\
\hline $80 / 40 \mathrm{mg}$ & $11(12.5)$ \\
\hline
\end{tabular}

a AZA: azathioprine, 6MP: 6 mercaptopurine, MTX: methotrexate, Cya: cyclosporine.

b Extra-intestinal manifestations.

c C-reactive protein.

found in the majority of patients (73.9\%). Concomitant medications at baseline included corticosteroids in 60 patients (68.2\%), immunosuppressants in 13 patients (14.8\%), and mesalazine in 49 patients, (55.7\%). Five patients $(5.7 \%)$ were not taking any medications. Seventy-seven patients $(87.5 \%)$ received an induction dose of ADA $160 \mathrm{mg}$ at week 0 and then $80 \mathrm{mg}$ at week 2, while 11 patients (12.5\%) received $80 \mathrm{mg}$ and $40 \mathrm{mg}$ at weeks 0 and 2 respectively.

All patients who showed clinical benefit from the induction regimen received maintenance treatment for at least 12 months. The median duration of ADA therapy was 13 months (IQR 6-21), with a median duration of follow-up of 15.5 months (IQR 12-24). Thirty-one patients (35.2\%) had their ADA dose increased to weekly. Among them, 13 patients (41.9\%) were able to de-escalate therapy after a median time of 5 months (IQR 3-9.5). However, none of the 9 patients who were dose-escalated early (between weeks 4 and 12) achieved clinical remission at week 12 . The main baseline characteristics of the patients are summarised in Table 1.

\subsection{Clinical effectiveness}

\subsubsection{Co-primary endpoints}

Fifteen of the 88 patients (17.0\%) were in clinical remission at week 4 . The rate of clinical remission increased through week 12 ( 25 of the 88 patients, $28.4 \%$ ), 24 (32 of the 88 patients, $36.4 \%$ ) and 54 (38 of the 88 patients, $43.2 \%$ ). No significant differences in clinical remission rates at different time points were observed between

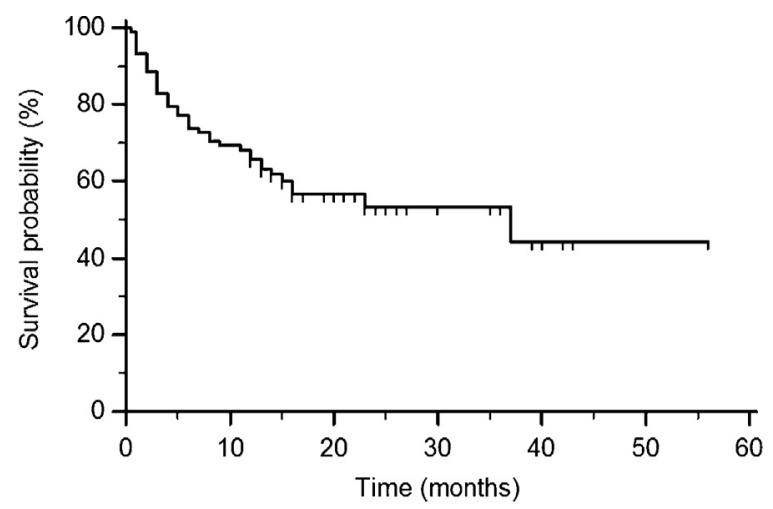

Number at risk

$\begin{array}{lllllll}88 & 61 & 23 & 8 & 3 & 1 & 1\end{array}$

Fig. 1. Cumulative probability of continuing adalimumab therapy during follow-up.

infliximab-naïve and infliximab-treated patients (week $4: 21.1 \%$ vs. $15.9 \%, p=0.8$; week 12 : $42.1 \%$ vs. $24.6 \%, p=0.2$; week $24: 36.8 \%$ vs. $36.2 \%, p=0.8$; week 54 : $47.4 \%$ vs. $43.2 \%, p=0.8$, respectively), although none of the 5 patients who experienced previous IFX primary failure achieved remission. The cumulative probability of continuing ADA therapy during follow up is shown in Fig. 1. After 12 months, the cumulative probability of continuing ADA was $65.9 \%$. Analysis of variance revealed a significant decrease in both the partial Mayo score $(p<0.001)$ and the CRP serum levels $(p<0.001)$ across different time points through week 54 . Post hoc analysis showed a significant decrease as early as week 4 (Fig. 2A and B).

\subsubsection{Secondary endpoints}

Sustained clinical remission, defined as clinical remission at week 12 maintained through weeks 24 and 54, was achieved in 15 of the 88 patients (17.04\%).

Sixty patients had active disease despite corticosteroids at baseline, with a daily median equivalent of prednisone dose of $25 \mathrm{mg}$ (IQR 15.0-37.5). Thirty-four of the 60 (56.7\%) were able to discontinue steroids and corticosteroid-free remission was achieved in 24 of the 60 patients $(40.0 \%)$ at week 54 . Analysis of variance revealed a significant daily prednisone equivalent dose reduction $(p<0.001)$ across different time points through week 54 and post hoc analysis identified a significant decrease as early as week 4 (Fig. 3).

A subgroup of 57 patients underwent endoscopy at baseline and after a median time of 11.1 months (IQR 5.1-13.2). Twentyeight of the 57 patients (49.1\%) achieved endoscopic remission and among them 15 of the 57 (26.3\%) achieved complete mucosal healing (Mayo endoscopic score $=0$ ).

Twenty-two of the 88 patients $(25.0 \%)$ underwent colectomy for failure of medical therapy, after a median time of 5.5
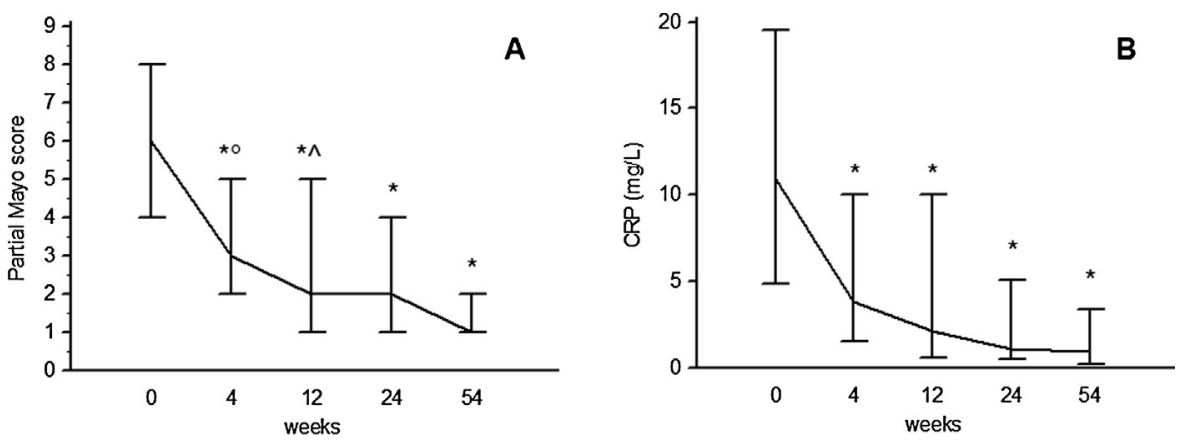

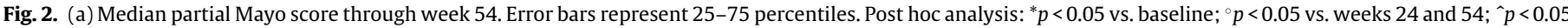

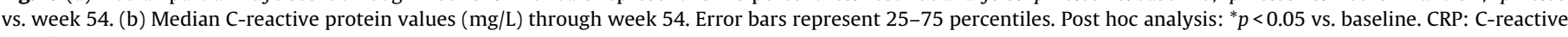
protein. 


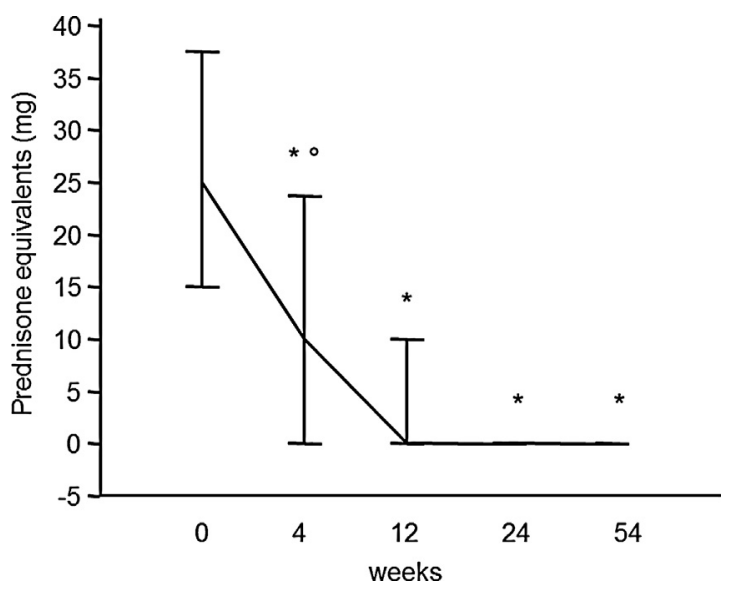

Fig. 3. Median daily prednisone equivalents dose $(\mathrm{mg})$ through week 54 . Error bars represent $25-75$ percentiles. ${ }^{*} p<0.05$ vs. baseline; ${ }^{\circ} p<0.05$ vs. weeks $12,24,54$.

months (IQR 3-13). The cumulative probability of colectomy during follow-up is shown in Fig. 4. The colectomy rate was higher in patients previously treated with infliximab compared with those who were infliximab-naïve, although the difference was not statistically significant ( $29 \%$ vs. $10.5 \%$ respectively, $p=0.1$ ).

Side effects were reported in 12 of the 88 patients (13.6\%): upper respiratory tract infection, community acquired pneumonia (2 patients), varicella zoster virus reactivation, DVT, dizziness, adalimumab intolerance, transient bilateral hearing loss, psoriasis, enteropathogenic Escherichia coli infection, atypical mycobacterial infection, pseudo erythrocytosis). ADA was discontinued in 6 of them $(6.8 \%)$.

\subsubsection{Predictors of clinical remission at 54 weeks and predictors of colectomy}

At univariate analysis, clinical remission at week 54 occurred more frequently in patients who achieved remission at week 12 and in patients with low CRP serum level at week 12 (76.0\% vs. $30.2 \%$; OR 4.17, 95\% CI 2.36-19.44; $p=0.0002$ and 66.7\% vs. 24.5\%; OR 2.63, 95\% CI 2.32-14.94; $p=0.0002$, respectively). Conversely, patients previously treated with immunosuppressants achieved a significantly lower remission rate at week 54 compared with patients never treated with immunosuppressants (33.8\% vs, 69.6\%; OR 0.67, $95 \%$ CI $0.08-0.66 ; p=0.0013$ ). No statistical significant differences were observed with regard to the other clinical variables considered. In the multivariate model, remission at week 12, CRP serum level at week 12 and previous immunosuppressant treatment were

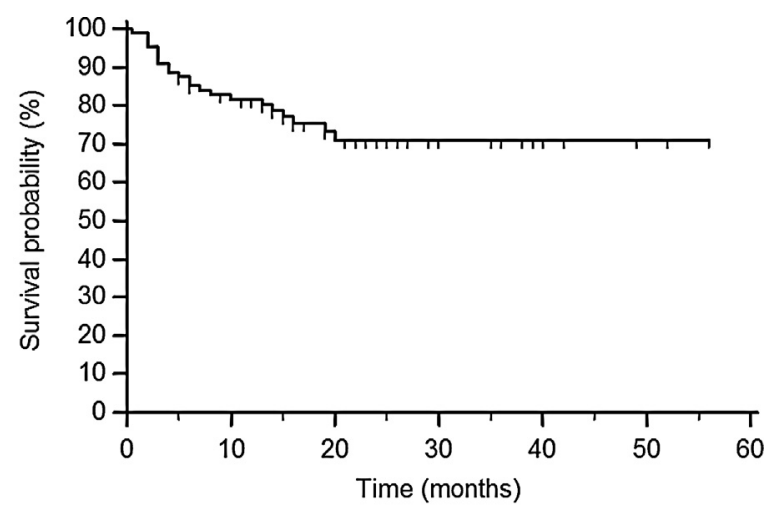

Number at risk

$\begin{array}{lllllll}88 & 69 & 30 & 10 & 5 & 2 & 1\end{array}$

Fig. 4. Cumulative probability of a course free of colectomy in the whole cohort.
Table 2

Predictors of clinical remission at week 54

\begin{tabular}{llll}
\hline Variable & OR & $95 \% \mathrm{CI}$ & $p$ value \\
\hline Sex & 1.65 & $0.55-4.93$ & 0.37 \\
Age (<median) & 1.70 & $0.56-5.12$ & 0.34 \\
Clinical remission at week 12 & 4.25 & $1.25-14.40$ & 0.02 \\
Low CRP a at week 12 (<median) & 5.19 & $1.67-16.10$ & 0.004 \\
Previous immunosuppressant use & 0.20 & $0.06-0.64$ & 0.007 \\
\hline a C-reactive protein. & & &
\end{tabular}

the only independent variables associated with the probability of clinical remission at 54 weeks (Table 2).

As far as the probability of colectomy is concerned, at univariate analysis previous use of immunosuppressants was the only clinical variable significantly associated with a higher rate of colectomy for the failure of medical therapy ( $95.5 \%$ vs. $4.5 \%$ of patients who never received immunosuppressants; HR 9.7, 95\% CI 1.46-9.07; $p=0.005$ ).

\section{Discussion}

This observational study is to our knowledge the largest case series of active UC treated with ADA in IBD referral centres in Italy. The vast majority of patients included were steroid-dependent or steroid-resistant, while in a minority of them the main indication was active UC with the presence of spondyloarthritis. More than $70 \%$ of patients had previously received conventional immunosuppressants and/or infliximab, mostly secondary failures or those who were intolerant. Therefore, this patient population may be considered a truly refractory and difficult-to-treat subgroup of UC.

Our results suggest that scheduled ADA is effective in this patient population. Approximately one third of patients entered into clinical remission within three months and this percentage increased to approximately $40 \%$ within one year, suggesting that, at least in this setting, ADA may be a slow-acting drug.

Among patients who were taking steroids at baseline, ADA has been shown to be a steroid sparing agent, allowing steroid withdrawal in more than $50 \%$ of patients and inducing steroid-free remission in $40 \%$ of them within one year.

Although less than $30 \%$ of patients achieved early remission and maintained sustained remission through one year, early remission, as well as an early drop in CRP values were independent predictors of probability of remission at one year. Conversely, the probability of remission at one year is reduced in patients with previous use of conventional immunosuppressants. This observation suggests that in a subgroup of patients in whom ADA shows a rapid impact on symptoms and systemic inflammation, a better outcome can be expected. As recently reported for moderate to severe steroid-dependent UC patients treated with infliximab [27], the observation that previous exposure to immunomodulators has a negative impact on long-term remission rates suggests that a more severe and refractory disease is less likely to benefit from ongoing conservative management. Nevertheless, previous infliximab exposure did not affect the long-term remission rate in our series: a possible explanation could be the low number of infliximab-naïve patients in this case series, who were mostly treated with ADA because of concomitant active spondyloarthritis.

As far as mucosal healing is concerned, the retrospective design of the study did not allow firm conclusions to be reached, mainly because endoscopy was performed according to individual clinical judgement and at different time points during follow up. Mucosal healing data were available in a subgroup of two thirds of patients of the entire cohort, in which endoscopy was performed at baseline and after a median follow-up of nearly one year. Results indicate that approximately half of the patients entered into endoscopic 
remission and more than one fourth achieved complete mucosal healing.

The cumulative probability of continuing ADA therapy over one year was $66 \%$. During the study period, according to clinical judgement, ADA dose escalation was necessary in one third of patients and more than $40 \%$ of them were able to de-escalate after a median of 5 months. However, the early need for dose escalation was not associated with the increased remission rate at week 12 .

The overall safety profile in this population was favourable. Adverse events leading to drug discontinuation were reported in less than $10 \%$ of patients.

During follow up 25\% of patients underwent colectomy because of primary failure or secondary loss of response to ADA therapy. In patients who required colectomy, surgery was performed after a median of 5.5 months (IQR 3-13). As for predictors of oneyear clinical remission, it is interesting to observe that the only clinical variable significantly associated with a higher rate of colectomy was previous conventional immunosuppressant exposure (HR 9.7, 95\% CI 1.46-9.07; $p=0.0055$ ). Previous infliximab exposure also increased the colectomy rate compared with infliximab-naïve status (29\% vs. $10.5 \%$ ), even though this difference did not reach statistical significance probably because of the low number of patients who ultimately required surgery and the low number of infliximab naïve patients enrolled in the study. Taken together, these observations suggest that those UC patients who have a refractory disease course and who have previously experienced multidrug treatment with poor response or intolerance are at a higher risk of a poor outcome.

The comparison between our results and those of RCTs [22,23] deserves some important consideration. In the ULTRA 1 and ULTRA 2 trials, the percentages of patients achieving remission at 8 weeks were $18.5 \%$ and $16.5 \%$ respectively. These figures are lower than the percentages of short-term remission observed in our study (12-week clinical remission: $28.4 \%$ ). Apart the different time point evaluations, the differences can be explained by different definitions of clinical remission, that in the two RCTs included endoscopic evaluation at week 8 (clinical remission defined as a total Mayo score $\leq 2$ points, with no individual subscore exceeding 1 point). The same consideration may be applied to the one-year remission rate, which was $17.3 \%$ in the ULTRA 2 study and $43.2 \%$ in our case series. Furthermore, in our experience, concomitant topical therapy with aminosalicylates was allowed and was used according to the physician's judgement. This might have had an influence on the better response in our population.

As far as long-term remission rates are concerned, another possible explanation can be the possibility of ADA dose escalation that was performed in more than one third of patients in our study. Nevertheless, additional analysis of the ULTRA 1 and ULTRA 2 studies showed that the proportion of patients in clinical remission, as assessed through the partial Mayo score, increased over time in ADA-treated patients, approaching 30\% between 8 and 16 weeks, a figure very close to our clinical remission rates at 12 weeks (28.4\%). This common finding suggests that the plateau of efficacy of ADA may have not yet been reached after 8 weeks; thus, longer exposure to ADA would probably be needed to observe a maximum response.

Several other open-label or retrospective observational studies addressing the use of ADA in patients with UC have been published [16-21]. All these studies had a small sample size (13-50 patients) and the results are difficult to compare because of differences in patient populations, follow-up, endpoints and definitions of response/remission. In the short term (4-12 weeks) a response rate of $25-80 \%$ and a remission rate of $5-27 \%$ have been reported. In the long-term (6-12 months), a response rate up to $50-70 \%$ has been reported. The colectomy rate ranges across studies from $0 \%$ to $46 \%$. Overall, our results are very similar to those reported by Taxonera et al. in a retrospective case series from Spain [19] in which 30 patients were enrolled. The 12-week remission rate was $26.7 \%$ and the probability of colectomy was $20 \%$. Moreover, clinical response at week 12 was predictive of a better long-term outcome.

In conclusion, in our "real-life" cohort of refractory and difficultto-treat UC patients, ADA has been shown to be effective. The achievement of clinical remission with a low CRP within 12 weeks and no experience of conventional immunosuppressants were the clinical characteristics associated with a better long-term outcome.

\section{Conflict of interest statement}

Alessandro Armuzzi received: consultancy from Abbott Laboratories, MSD; lecture fees from Abbott Laboratories, MSD, Chiesi, Ferring, Nycomed, Otsuka; educational grants from Abbott Laboratories, MSD, Ferring, Nycomed.

Marco Daperno: received consultancy from Abbott Laboratories, MSD, Chiesi; lecture fees from Abbott Laboratories, MSD, Chiesi, Sofar.

Sandro Ardizzone received financial sponsorship from Abbott Laboratories, MSD, Nycomed.

Fabrizio Bossa received financial sponsorship from Abbott Laboratories, MSD.

Fabiana Castiglione received financial sponsorship from Abbott Laboratories, MSD, Chiesi.

Silvio Danese: has served as a speaker, consultant and advisory board member for Abbott Laboratories, Merck \& Co/Schering Plough, UCB Pharma, Ferring, Cellerix, Millenium Takeda, Nycomed, Pharmacosmos, Actelion, Alphawasserman, Genentech, Pfizer, Astra Zeneca, Novo Nordisk, Cosmo Pharmaceuticals, Vifor, Johnson and Johnson.

Renata D'Incà received financial sponsorship from Abbott Laboratories, MSD, Chiesi.

Walter Fries received: research grant and advisory board fees from Schering-Plough/MSD; fees continuing education events from MSD and Sofar.

Paolo Gionchetti received lecture and consultancy fees from Abbott Laboratories, MSD, Chiesi.

Ambrogio Orlando received consultancy/lecture fees/educational grants from Abbott Laboratories, MSD, Nycomed Chiesi, Sofar.

Fernando Rizzello received: consultancy/lecture fees/educational grants from Abbott Laboratories, MSD, Chiesi, Ferring.

Luisa Guidi received financial sponsorship from Abbott Laboratories, MSD.

Claudio Papi received: consultancy from Abbott Laboratories, MSD; lecture fees from Chiesi, Sofar.

Livia Biancone, Alessandra Coli, Daniela Pugliese, Vito Annese, Annalisa Aratari, Paola Balestrieri, Maria Cappello, Michele Cicala, Pietro Dulbecco, Giuseppe Feliciangeli, Stefania Genise, Stefano Gozzi, Anna Kohn, Roberto Lorenzetti, Monica Milla, Sara Onali, Luigi Giovanni Papparella, Sara Renna, Chiara Ricci, Raffaello Sostegni: none declared.

All Authors have received no payment in the preparation of this manuscript.

\section{Appendix A. Co-Author affiliations:}

Gastroenterology Unit, Department of Internal Medicine, "Tor Vergata" University, Rome, Italy (Livia Biancone, Sara Onali); S.C. Gastroenterologia, Azienda Ospedaliera Ordine Mauriziano, Torino, Italy (Marco Daperno, Raffaello Sostegni); Internal Medicine, Emergency Department, University of Pisa, Italy (Alessandra Coli); Division of Gastroenterology, Department of Medical \& Surgical Sciences, University Hospital Careggi, Florence, Italy (Vito Annese, Stefania Genise, Monica Milla); UOC Gastroenterologia \& Epatologia, S. Filippo Neri Hospital, Rome, Italy (Annalisa Aratari, Claudio 
Papi); IBD Unit, L. Sacco Hospital, Milan, Italy (Sandro Ardizzone); Department of Digestive Disease, University Campus Bio Medico, Rome, Italy (Paola Balestrieri, Michele Cicala); Gastroenterology Unit, Casa Sollievo della Sofferenza, San Giovanni Rotondo, Italy (Fabrizio Bossa); Gastroenterology Section, Di. Bi. Mis., University of Palermo, Italy (Maria Cappello); Gastroenterology, Department of Clinical and Experimental Medicine, University "Federico II" of Naples, Italy (Fabiana Castiglione); IBD Unit, Istituto Clinico Humanitas, Rozzano, Italy (Silvio Danese); Department of Surgical, Oncological and Gastroenterological Sciences, University of Padua, Italy (Renata D'Incà); GI Unit, Department of Internal Medicine, University of Genoa, Italy (Pietro Dulbecco); Gastroenterology Unit, Macerata Hospital, Macerata, Italy (Giuseppe Feliciangeli); Clinical Unit for Chronic Bowel Disorders, Department of Clinical and Experimental Medicine, University of Messina, Italy (Walter Fries); I Clinica Medica e Terapia Medica, Department of Internal Medicine and Gastroenterology, University of Bologna, Italy (Paolo Gionchetti, Fernando Rizzello); Gastroenterology Unit, San Donato Hospital, Arezzo, Italy (Stefano Gozzi); Gastroenterology Unit, San Camillo-Forlanini Hospital, Rome, Italy (Anna Kohn, Luigi Giovanni Papparella); Gastroenterology Unit, PTP Nuovo Regina Margherita, Rome, Italy (Roberto Lorenzetti); Villa Sofia-V. Cervello Hospital, Department of Medicine, University of Palermo, Italy (Ambrogio Orlando, Sara Renna); Gastroenterology Unit, University of Brescia, Italy (Chiara Ricci).

\section{References}

[1] Edwards FC, Truelove SC. The course and prognosis of ulcerative colitis. Gut 1963;4:299-315.

[2] Ng SC, Kamm MA. Therapeutic strategies for the management of ulcerative colitis. Inflammatory Bowel Diseases 2009;15:935-50.

[3] Danese S, Fiocchi C. Ulcerative colitis. New England Journal of Medicine 2011;365:1713-25.

[4] Rutgeerts P, Sandborn WJ, Feagan B, et al. Infliximab for induction and maintenance therapy for ulcerative colitis. New England Journal of Medicine 2005;233:2462-73.

[5] Järnerot G, Hertervig E, Friis-Liby I, et al. Infliximab as rescue therapy in severe to moderately severe ulcerative colitis: a randomized, placebo-controlled study. Gastroenterology 2005;128:1805-11.

[6] Sandborn WJ, Rutgeerts P, Feagan BG, et al. Colectomy rate comparison after treatment of ulcerative colitis with placebo or infliximab. Gastroenterology 2009;137:1250-60.

[7] Colombel JF, Rutgeerts P, Reinisch W, et al. Early mucosal healing with infliximab is associated with improved long-term clinical outcomes in ulcerative colitis. Gastroenterology 2011;141:1194-201.

[8] Ferrante M, Vermeire S, Fidder H, et al. Long-term outcome after infliximab for refractory ulcerative colitis. Journal of Crohn's and Colitis 2008;2:219-25.

[9] Orlando A, Armuzzi A, Papi C, et al. The Italian Society of Gastroenterology (SIGE) and the Italian Group for the study of Inflammatory Bowel Disease
(IG-IBD) Clinical Practice Guidelines: the use of tumor necrosis factor-alpha antagonist therapy in inflammatory bowel disease. Digestive and Liver Disease 2011;43:1-20.

[10] Hanauer SB, Wagner CL, Bala M, et al. Incidence and importance of antibody responses to infliximab after maintenance or episodic treatment in Crohn's disease. Clinical Gastroenterology and Hepatology 2004;2:542-53.

[11] HUMIRA ${ }^{\circledR}$ Product Monograph. Abbott.

[12] Hanauer SB, Sandborn WJ, Rutgeerts P, et al. Human anti-tumor necrosis factor monoclonal antibody (adalimumab) in Crohn's disease: the CLASSIC-I trial. Gastroenterology 2006;130:323-33.

[13] Colombel JF, Sandborn WJ, Rutgeerts P, et al. Adalimumab for maintenance of clinical response and remission in patients with Crohn's disease: the CHARM trial. Gastroenterology 2007;132:52-65.

[14] Sandborn WJ, Rutgeerts P, Enns R, et al. Adalimumab induction therapy for Crohn disease previously treated with infliximab: a randomized trial. Annals of Internal Medicine 2007;146:829-38.

[15] Panaccione R, Colombel JF, Sandborn WJ, et al. Adalimumab sustains clinical remission and overall clinical benefit after 2 years of therapy for Crohn's disease. Alimentary Pharmacology and Therapeutics 2010;31:1296-309.

[16] Oussalah A, Laclotte C, Chevaux JB, et al. Long-term outcome of adalimumab therapy for ulcerative colitis with intolerance or lost response to infliximab: a single-centre experience. Alimentary Pharmacology and Therapeutics 2008;28:966-72.

[17] Afif W, Leighton JA, Hanauer SB, et al. Open-label study of adalimumab in patients with ulcerative colitis including those with prior loss of response or intolerance to infliximab. Inflammatory Bowel Diseases 2009;15: 1302-7.

[18] Gies N, Kroeker KI, Wong K, et al. Treatment of ulcerative colitis with adalimumab or infliximab: long-term follow-up of a single-centre cohort. Alimentary Pharmacology and Therapeutics 2010;32:522-8.

[19] Taxonera C, Estellés J, Blanco I, et al. Adalimumab induction and maintenance therapy for patients with ulcerative colitis previously treated with infliximab. Alimentary Pharmacology and Therapeutics 2011;33:340-8.

[20] Ferrante M, Karmiris K, Compernolle G, et al. Efficacy of adalimumab in patients with ulcerative colitis: restoration of serum levels after dose escalation results in a better long-term outcome. Gut 2011;60(Suppl. 3):A72.

[21] McDermott E, Murphy S, Keegan D, et al. Efficacy of adalimumab as a long term maintenance therapy in ulcerative colitis. Journal of Crohn's and Colitis 2013;7:150-3.

[22] Reinisch W, Sandborn WJ, Hommes DW, et al. Adalimumab for induction of clinical remission in moderately to severely active ulcerative colitis: results of a randomised controlled trial. Gut 2011;60:780-7.

[23] Sandborn WJ, van Assche G, Reinisch W, et al. Adalimumab induces and maintains clinical remission in patients with moderate-to-severe ulcerative colitis. Gastroenterology 2012;142:257-65.

[24] Dignass A, Eliakim R, Magro F, et al. Second European evidence-based Consensus on the diagnosis and management of ulcerative colitis: definition and diagnosis. Journal of Crohn's and Colitis 2012;6:965-90.

[25] Silverberg MS, Satsangi J, Ahmad T, et al. Toward an integrated clinical, molecular and serological classification of inflammatory bowel disease: report of a Working Party of the 2005 Montreal World Congress of Gastroenterology. Canadian Journal of Gastroenterology 2005;19(Suppl. A):5-36.

[26] Schroeder KW, Tremaine WJ, Ilstrup DM. Coated oral 5-aminosalicylic acid therapy for mildly to moderately active ulcerative colitis. A randomized study. New England Journal of Medicine 1987;317:1625-9.

[27] Armuzzi A, Pugliese D, Danese S, et al. Infliximab in steroid-dependent ulcerative colitis: effectiveness and predictors of clinical and endoscopic remission. Inflammatory Bowel Disease 2013;19:1065-72. 